\title{
Multiple concurrent feedforward and feedback streams in a cortical
}

\author{
hierarchy
}

\author{
Elham Barzegaran*, Gijs Plomp*
}

Perceptual Networks Group, Department of Psychology, University of Fribourg, Rue de

Faucigny 2, 1700 Fribourg, Switzerland

\section{*Corresponding Authors:}

Elham Barzegaran, e.barzegaran@gmail.com

Gijs Plomp, gijs.plomp@unifr.ch 


\section{Abstract}

2 Visual stimuli evoke fast-evolving activity patterns that are distributed across multiple cortical

3 areas. These areas are hierarchically structured, as indicated by their anatomical projections, but

4 how large-scale feedforward and feedback streams are functionally organized in this system

5 remains an important missing clue to understanding cortical processing. By analyzing visual

6 evoked responses in laminar recordings from six cortical areas in awake mice, we established the

7 simultaneous presence of two feedforward and two feedback networks, each with a distinct

8 laminar functional connectivity profile, frequency spectrum, temporal dynamics and functional

9 hierarchy. We furthermore identified a distinct role for each of these four processing streams, by

10 leveraging stimulus contrast effects and analyzing receptive field convergency along functional

11 interactions. Our results support a dynamic dual counterstream view of hierarchical processing

12 and provide new insight into how separate functional streams can simultaneously and

13 dynamically operate in visual cortex. 


\section{Introduction}

2 Visual processes exhibit complex patterns of fast-evolving activity that are distributed across

3 cortex. Within $100 \mathrm{~ms}$ after stimulus onset, activity spreads throughout visual cortex and beyond,

4 both in primates and rodents ${ }^{1,2}$, enabling functional cortico-cortical interactions that are

5 necessary for even the most elementary visual operations ${ }^{3,4}$. Such networked processing occurs

6 over dense anatomical projections that reciprocally connect cortical areas. Across visual cortex,

7 the structure of projections indicates a hierarchical organization, with feedforward projections

8 that can propagate sensory activity from lower to higher-level areas, and feedback projections

9 that can exert downstream influences ${ }^{5-7}$. Understanding how visual cortex enables fast,

10 distributed processing over its fixed hierarchical structure may provide important clues to further

11 understanding large-scale cortical function.

12 Visual processing critically depends on both feedforward and feedback processes ${ }^{8}$. Based on

13 onset latencies after stimulation, it was shown that the feedforward spread of activity from V1

14 mostly follows the structurally defined hierarchy ${ }^{1,2}$, and that a fast feedforward sweep precedes

15 feedback signals from higher order areas ${ }^{9}$. But due to the fast mixing of activities after onset, it

16 remains unclear how feedforward and feedback interactions dynamically shape visual

17 processing, which mostly occurs after activity onset.

18 Candidate mechanisms for how visual cortex could support a mixture of feedforward and

19 feedback processes have been proposed based on the laminar structure of hierarchical

20 projections, and the rhythmicity of neural activity. Recordings across cortical columns have

21 indicated laminar and frequency differences in feedforward and feedback activities ${ }^{4,10}$, where

22 gamma activity in supragranular layers is thought of as feedforward propagation ${ }^{10-13}$, and lower- 
1 frequency activity in infragranular layers is associated with inter-areal feedback ${ }^{14,15}$. But

2 although quasi-periodic activity is pervasive in neural systems and much studied, there is no

3 strong consensus on the functional role of different frequency bands ${ }^{15-17}$. In addition, scale-free

4 activity without a dominant rhythm is an equally widespread phenomenon in neural systems ${ }^{18,19}$.

5 To functionally characterize feedforward and feedback streams in the time and frequency

6 domain, we used visually-evoked activity recorded in awake mice across the visual hierarchy

7 with laminar resolution. By using parallel factor analysis of directed cortico-cortical networks,

8 we uncovered the simultaneous presence of two feedforward and two feedback networks, each

9 with distinct laminar connectivity profiles, operational frequencies, temporal dynamics and

10 functional hierarchical organization. We could furthermore determine distinct roles for each of

11 these four processing streams, by leveraging well-described effects of stimulus contrast and by

12 analyzing receptive field (RF) convergency ${ }^{20,21}$. 


\section{Results}

\section{Evoked activity and functional interactions show complex dynamics}

3 We analyzed local field potentials (LFP) from 11 awake wild-type mice recorded simultaneously

4 from 5 or 6 visual areas with laminar resolution during presentation of high or low contrast

5 drifting gratings (Figure 1a-b). These data were made available by the Allen Brain Institute (see

6 Methods). Stimulus-evoked LFPs showed expected fast dynamic responses across layers and

7 areas throughout the cortical hierarchy (Figure 1c) ${ }^{2}$, in line with results from non-human

8 primates ${ }^{1}$.

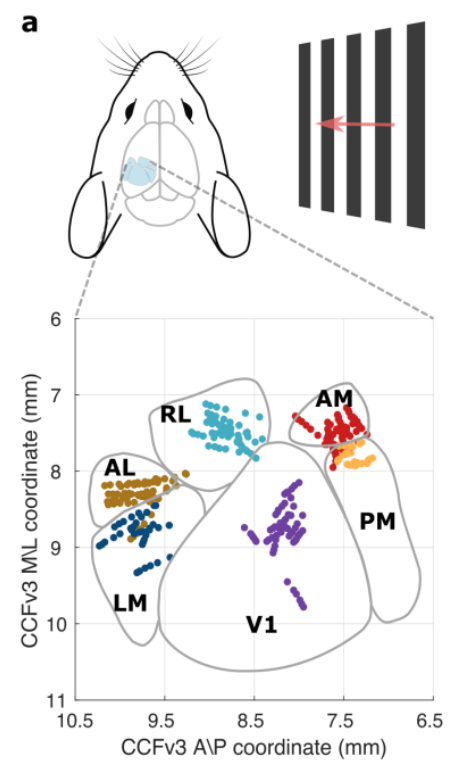

b

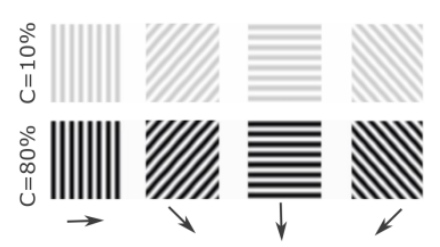

C
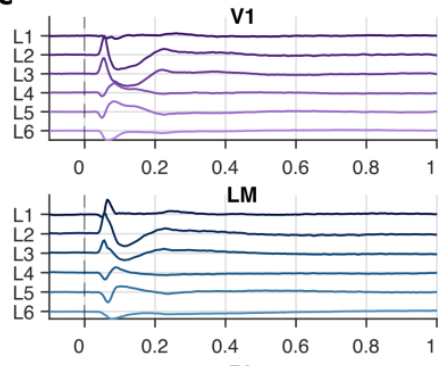

RL
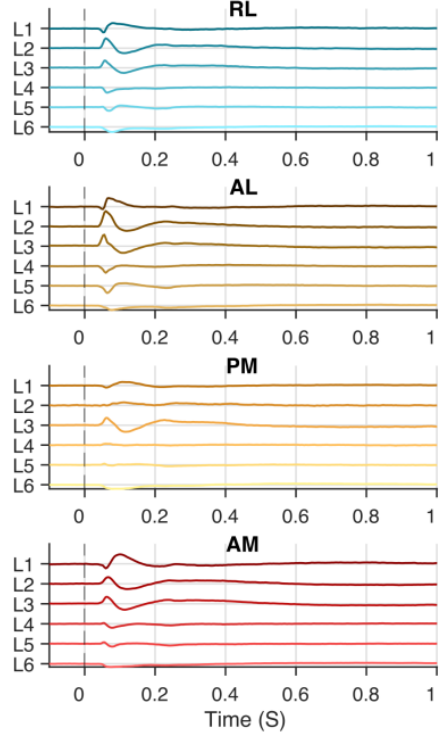

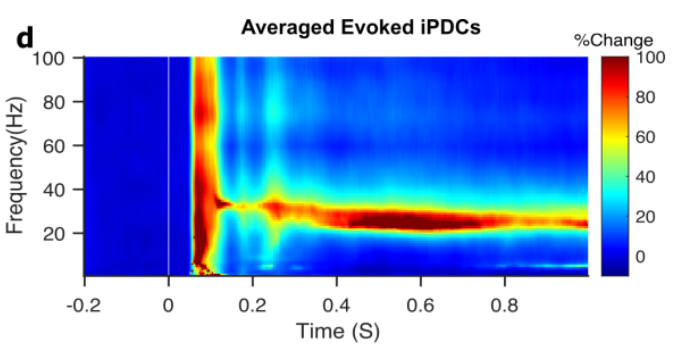

e Evoked Between Area iPDCs
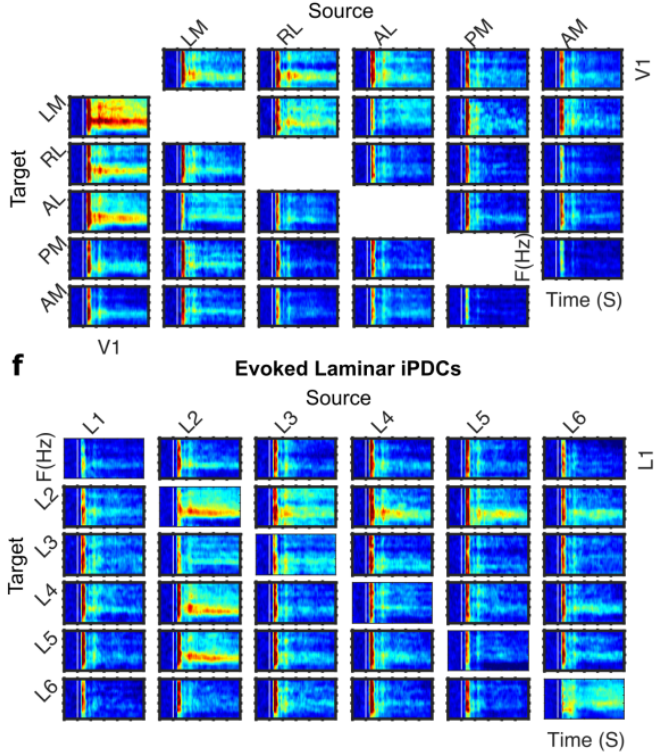

Figure 1. Visual stimuli evoke large-scale activity and functional interactions. a, Laminar recording sites across

11 cortical areas from 11 mice, in Allen brain institute's standard CCFv3 space. b, Visual stimuli were drifting gratings 
1 of high $(80 \%)$ or low $(10 \%)$ contrast, presented for 2 S. c, Grand-average bipolar LFPs evoked by high contrast

2 stimuli, for L1 to L6 in six visual areas, shown in anatomical hierarchical order ${ }^{7}$. d, Directed functional connectivity

3 (iPDC) in the time and frequency domain, averaged over all areas, layers, and animals. e, Time-frequency

4 connectivity between areas, averaged over layers and animals. f, Time-frequency connectivity between layers,

5 averaged over areas and animals. Heatmaps show percentage of post-stimulus change, columns indicate sources and

6 rows indicate targets of directed functional interactions.

7 To derive functional interaction strengths between all areas and layers, we used an optimized

8 Kalman filter to model dependencies between LFPs and calculated the information Partial

9 Directed Coherence (iPDC) ${ }^{22,23}$. iPDC provides a multivariate measure of directed functional

10 connectivity (Granger causality) with high temporal and frequency resolution. The resulting

11 connectivity matrices revealed a detailed time-frequency representation of between-area

12 interaction strengths across visual cortex, with laminar resolution (Figure 1d-f).

13 Averaging across areas and layers, we found that stimulus onset evoked statistically robust

14 functional connectivity, and that the interactions were contrast-sensitive. Evoked connectivity

15 onset was around $50 \mathrm{~ms}$ for high contrast, and $85 \mathrm{~ms}$ for low contrast stimuli (Figure 2),

16 following known contrast dependency of LFPs ${ }^{20}$. The initial response was followed by

17 sustained, frequency-specific increases in gamma band interactions that peaked around 35 and 25

$18 \mathrm{~Hz}$ for high and low contrast stimuli respectively. This indicates that reducing stimulus contrast

19 lowers the gamma band interaction frequency, similar to its effect on LFP power spectra ${ }^{16,24,25}$. 
1

2
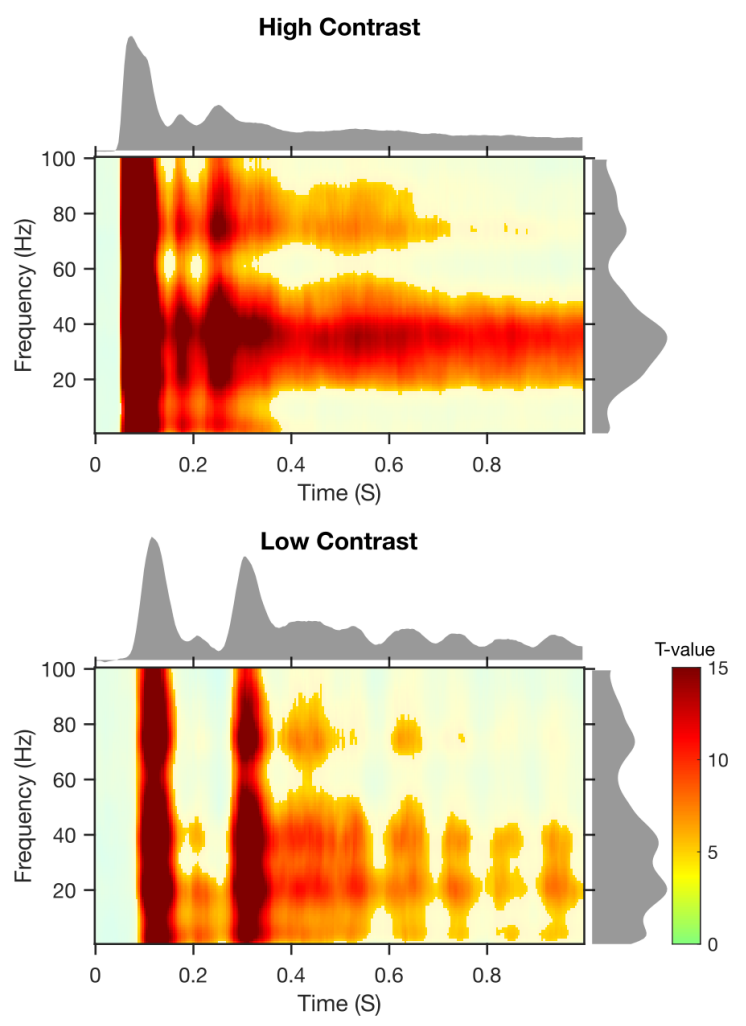

Figure 2. Visually-evoked functional connectivity for high and low contrast stimuli. Time-frequency plots show statistically significant functional connectivity increases with respect to baseline (one-way t-tests $p<0.05$, Bonferroni corrected), marginal distributions reflect t-values. Data averaged across layers and areas.

\section{Four concurrent networks in mouse visual cortex}

To further analyze the time- and frequency-resolved connectivity across visual cortex, we used Parallel Factor Analysis (PARAFAC), a tensor rank decomposition method that provides robust and interpretable results ${ }^{26}$. PARAFAC has previously been used to identify constituent components of time-varying power spectral density (PSD) ${ }^{27,28}$ and functional connectivity ${ }^{29}$. PARAFAC guarantees a unique solution when the appropriate number of components is selected. To select this number, we combined multiple criteria, including the core consistency diagnostic, mean square error, model convergence, and visual inspection ${ }^{26,28,30}$. This showed that 
1 the functional connectivity matrices were best decomposed into four components, independently

2 for low and high contrast conditions, and explaining around 90\% of total variance. We confirmed

3 the reliability and validity of the four-way model across animals using a bootstrap analysis that

4 showed high consistency across bootstraps for each component (average correlation coefficients

5 for all components $>0.81, p<0.05$ ), and low correlations between components (average

6 correlation coefficients $=0.21$, not significant).

7 This way, we established that the dynamic laminar functional connectivity pattern across the

8 visual hierarchy can be decomposed into four distinct networks, each of which reflects a

9 processing stream defined by its directed connectivity strengths between areas over source

10 layers, target layers, frequency spectrum and temporal dynamics (Figure 3). 
a
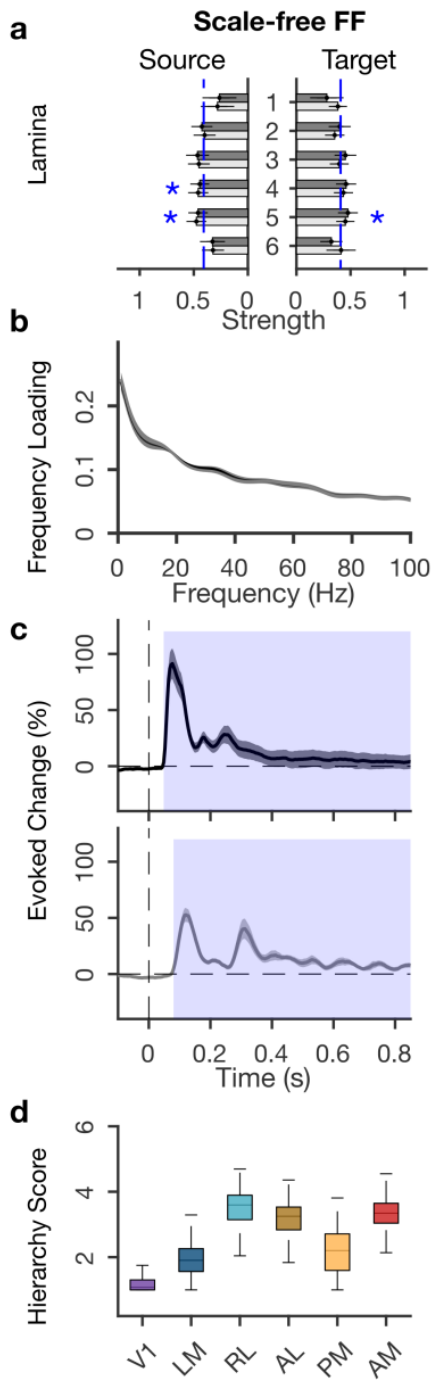
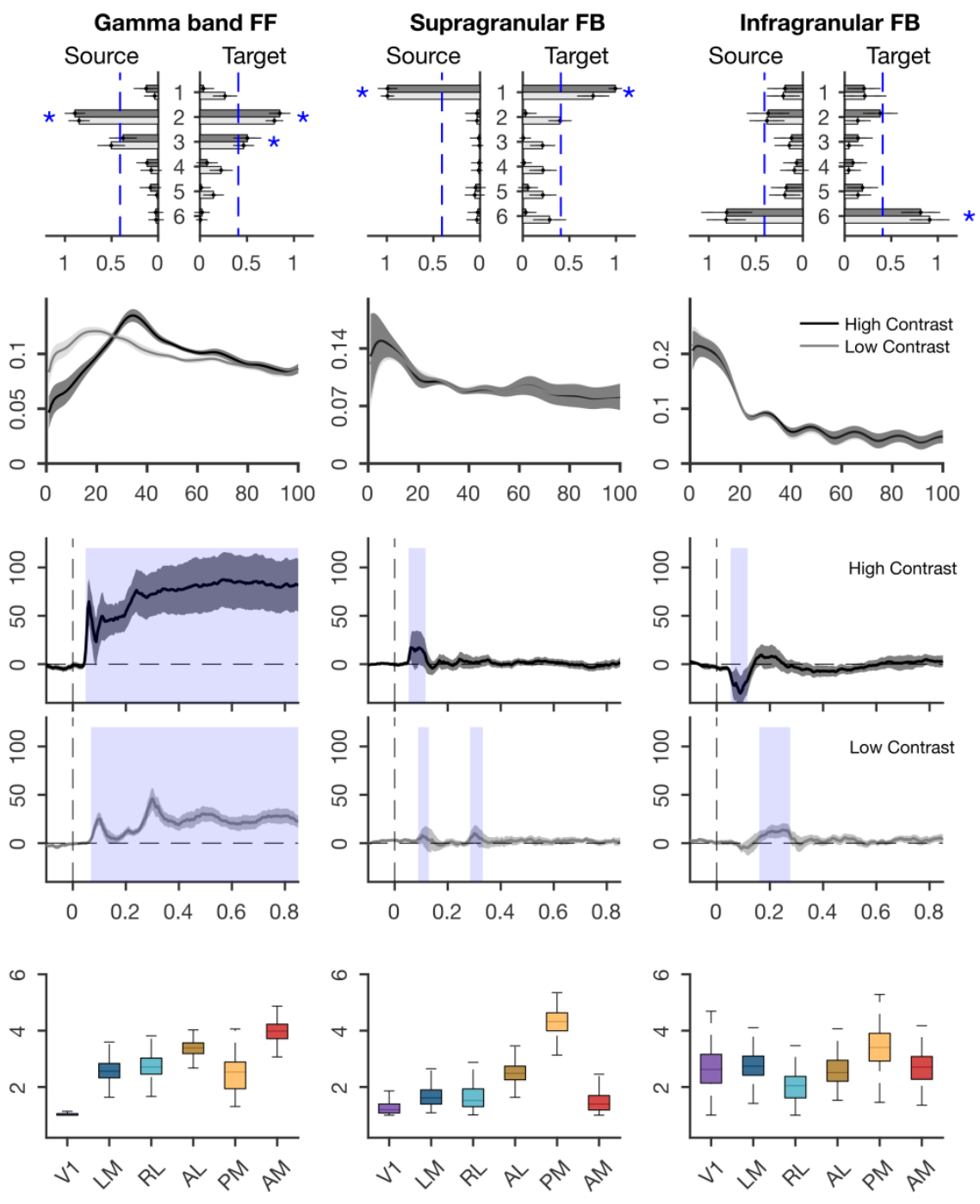

Significant evoked response 
bootstrapped 25 and 75 quantiles of the hierarchy scores, whiskers indicate extreme values excluding outliers. For a, $\mathrm{b}$, and $\mathrm{c}$ error bars and grey shading indicate standard deviations over bootstraps $(\mathrm{n}=500)$.

\section{$4 \quad$ A dominant scale-free network}

5 We found that a scale-free network was the dominant network, accounting for about $50 \pm 5 \%$

6 (mean \pm s.d.) of the PARAFAC model amplitude, in both stimulus contrasts. The connectivity

7 strengths in this network were relatively uniformly distributed over source and target layers, but

8 showed significantly stronger connections from L4 and L5, and toward L5 than expected by

9 chance (Figure 3a). This pattern of laminar connectivity resembles the distribution of

10 feedforward targeting neurons through infragranular layers, particularly L5, as established by

11 retrograde tracing in mouse ${ }^{7}$ and monkey ${ }^{6}$.

12 The network's frequency spectrum followed a power-law distribution for both low and high

13 contrast stimuli, revealing a scale-free temporal dynamic where no particular rhythm dominates

14 the functional interactions (Figure $3 \mathrm{~b}$ ). Power-law distributions take the form $1 / \mathrm{f}^{\beta}$, where $\beta$

15 quantifies the self-similarity in the time domain (autocorrelation). Model fitting of the scale-free

16 network showed a $\beta$ exponent of 0.4 , lower than typical autocorrelations for electrophysiological

17 data, which fall in the range of $1-3$ for the frequency range of $1-100 \mathrm{~Hz}{ }^{19}$. To test whether the

18 low $\beta$ values are characteristic of the network or of the underlying LFPs, we fitted power law

19 distributions to the PSDs of LFPs in all areas. This revealed higher $\beta$ exponents $(2 \pm 0.5$ mean \pm

20 s.d. over bootstraps), demonstrating that low self-similarity rather is a property of the scale-free

21 network than of the underlying LFPs. These results indicate that between-area functional

22 interactions have greater agility in time than the within-area activity. 
1 The scale-free network showed robust amplitude increases quickly after stimulus onset, which

2 decreased after around $150 \mathrm{~ms}$, but exceeded pre-stimulus values throughout the epoch $(p<0.05$,

3 Bonferroni corrected, Figure 3c). This fast network activation, together with its laminar profile

4 supports the idea that sensory activation is quickly relayed across areas over infragranular layers

$5 \quad 31$. The scale-free network showed different dynamics depending on stimulus contrast. With low

6 contrast, onset latencies were delayed (from around 50 to $80 \mathrm{~ms}$ ) and a more pronounced second

7 peak occurred at around $300 \mathrm{~ms}$ before amplitudes decreased (c.f. Figure 2).

8 To characterize whether the direction of interactions followed a predominant feedforward or

9 feedback pattern, we derived functional hierarchy scores from between-area connectivity

10 strengths ${ }^{12}$ (see Methods). This revealed a hierarchical organization with V1 and LM at the

11 bottom followed by the other areas (Figure 3d), indicating a mainly feedforward direction of

12 interactions. The functional hierarchy resembled the structural hierarchy established from axonal

13 projections ${ }^{2,7}$, but it put PM at a lower ordinal position. Area PM, like V1, preferentially

14 responds to low temporal and high spatial frequencies, while LM, AL, RL, and AM prefer high

15 temporal and low spatial frequencies ${ }^{32}$. The temporal and spatial frequencies of the drifting

16 gratings were closer to the preferred frequencies of PM, allowing it to more strongly drive

17 activity in other areas, thus lowering its position in the functional hierarchy.

18 In summary, we found a dominant scale-free network whose laminar connectivity profile, and

19 functional hierarchy indicate a predominant feedforward direction of interactions. The network

20 responds strongly but transiently to stimulus onsets with some contrast sensitivity.

\section{Visual stimuli recruit a feedforward gamma band network}


1 The second strongest network contributed $18 \pm 2 \%$ of total model amplitude, for both stimulus

2 contrast. Its laminar connectivity pattern showed L2 and L3 as both the strongest source and

3 target layers (Figure 3a), a pattern that is typically associated with feedforward processing

4 streams in mouse and primate ${ }^{6,33-35}$.

5 This network showed clear peak amplitudes in the gamma band, broadly defined as 25-100 Hz.

6 Visually induced gamma band activity is typically linked to feedforward processes ${ }^{11,24}$ and may

7 help synchronize activities between cortical regions ${ }^{13}$. The network's peak frequency changed

8 with stimulus contrast, centering around $38 \mathrm{~Hz}$ for high contrast and around $26 \mathrm{~Hz}$ for low

9 contrast (Figure 3b), resembling the frequency shift observed in overall functional connectivity

10 strengths (Figure 2), and the contrast sensitivity of gamma band LFP responses ${ }^{16,25}$. Such strong

11 contrast-sensitivity indicates a role in processing stimulus content.

12 The gamma band network also showed contrast-dependency in its temporal dynamics (Figure

13 3c). For high contrast stimuli, this network showed increased amplitudes after $50 \mathrm{~ms}$ that were

14 sustained throughout stimulus presentation. In the low contrast condition, amplitude increase

15 started around $70 \mathrm{~ms}$, and were sustained with an apparent rhythmic modulation. These effects

16 resemble the contrast effects in overall functional connectivity and those in the scale-free

17 network.

18 Functional hierarchy analysis confirmed the feedforward character, with V1 located lowest and

19 the ordinal position of the other areas following the structural hierarchy ${ }^{7,34}$. As in the scale-free

20 network, the gamma band network showed a relatively low hierarchical position for area PM,

21 possibly due to the stimulus characteristics and response properties of this area ${ }^{32}$. 
1 In sum, the gamma band network shows a laminar inter-areal connectivity pattern and

2 hierarchical organization that conforms to known feedforward structural connectivity patterns.

3 The network showed a sustained response to visual stimulation that strongly depended on

4 stimulus contrast.

\section{$5 \quad$ Two low-frequency feedback networks}

6 In addition to the feedforward networks, we uncovered two feedback networks that accounted for

$7 \quad 17 \pm 2 \%$ and $15 \pm 4 \%$ of model amplitude. One was a supragranular network, with putative L1 as

8 the main source and target of inter-areal connections, the other was an infragranular network

9 with L6 as the main source and target (Figure 3a). Anatomical studies of primate visual cortex in

10 monkey established that besides the well-known feedback pathway over L6, another feedback

11 pathway exists over supragranular layers L1 and L2 6,34,35. Our findings for the first time

12 distinguish these two feedback pathways using analysis of functional data and demonstrate their

13 presence in mouse.

14 In accord with a presumed feedback function, we found that both networks showed a broad low-

15 frequency distribution, which peaked at 5 and $6 \mathrm{~Hz}$ respectively and included the alpha band

16 (full-width half-maximum of 1-14 Hz and 2.5-15.5 Hz respectively). Previous works identified

17 low-frequency interactions as signatures of feedback processing in visual cortex of cat and

18 monkey, using functional connectivity analysis and causal interference ${ }^{10,36}$.

19 The two feedback networks showed strikingly different evoked responses and contrast

20 sensitivities. The supragranular feedback network showed fast and transient evoked amplitude

21 increases with typical contrast sensitivity, i.e. slower onset and reduced amplitudes for low

22 contrast stimuli (50 vs. $85 \mathrm{~ms}$ ). In addition, low contrast stimuli evoked a second amplitude 
1 increase at around the same latency that the feedforward networks showed a second low-contrast

2 peak. The infragranular feedback network, conversely, showed quickly decreased amplitudes for

3 high contrast stimuli, between 50 and $120 \mathrm{~ms}$, but increased amplitudes for low contrast stimuli

4 at longer latencies, between 155 and $280 \mathrm{~ms}$. This indicates enhanced feedback for low contrast

5 stimuli at longer latencies 9,21. In addition, these findings show how stimulus contrast

6 differentially recruits the two feedback pathways, first over infragranular and then over

7 supragranular pathways, suggesting a functional dissociation of feedback streams.

8 The feedback networks had distinct functional hierarchies. The supragranular network showed

$9 \mathrm{AM}$ and V1 at the bottom of a hierarchical organization that otherwise resembled the structural

10 hierarchy 2,7 , but with lower hierarchy scores for RL and AL than in the feedforward networks.

11 The low hierarchical positions of high-level visual areas characterize the supragranular network

12 as a feedback network, in line with its laminar connectivity and frequency spectrum. For the

13 infragranular network, area RL showed the lowest hierarchy score, but the other areas appeared

14 not to be hierarchically ordered, with V1 having a higher hierarchy score than in any of the other

15 networks. The low RL position supports a predominant feedback directionality for the

16 infragranular network.

17 Taken together, these findings distinguish two feedback networks, one operating over

18 supragranular and one over infragranular layers. The networks share a low-frequency profile but

19 differ in how they respond to stimulus onset, their contrast sensitivity, and their functional

20 hierarchies. This differentiation is in line with the distinct influences of feedback arriving in the

21 superficial or deep layers, and how this affects neural activity in the cortical column ${ }^{31,37-39}$.

\section{Rhythmic modulation of feedforward networks}


1 The feedforward networks appeared to show rhythmic amplitude modulations in time, especially

2 for low contrast stimuli (Figure 3). To investigate this, we applied Fourier transformation on the

3 post-stimulus window of temporal loadings, after removing linear trends. This revealed a

4 rhythmic modulation of both feedforward networks that was most prominent for low contrast

5 stimuli and had a peak frequency of around $5 \mathrm{~Hz}$ (Figure 4).

6 Theta-band rhythms in mice are common in hippocampus, but also occur in sensory areas ${ }^{15,40}$. In

7 the current data, the theta modulation is likely of cortical origin because it varies with stimulus

8 contrast. The possibility that micro-saccades account for the theta modulation of feedforward

9 networks was excluded in a control analysis (data not shown). Theta rhythms in sensory cortex

10 have been shown to organize local activity, in mouse and monkey ${ }^{40-42}$. Although theta-rhythmic

11 functional interactions were previously reported in monkey ${ }^{43}$ and cat ${ }^{14}$, our results show a 12 rhythmic amplification of entire feedforward networks, particularly when stimuli are less 13 visible.

14

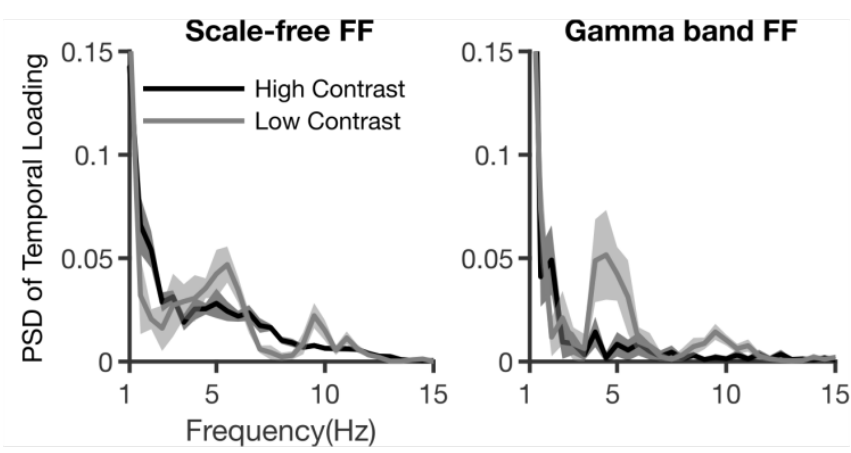

15 Figure 4. Spectral power of feedforward network dynamics. Fourier transformation of temporal dynamics for 16 feedforward networks, for two contrast conditions. Shaded area indicates standard deviation over bootstraps. 


\section{Receptive field distances and functional connectivity}

2 Considerable evidence shows that feedforward projections tend to converge onto matching

3 receptive fields $(\mathrm{RF})$ in upstream areas, whereas feedback projections tend to be more divergent

$4 \quad 6,8,44$. Convergent and divergent projections play different roles in spatial processing, and we

5 therefore asked how inter-areal functional connectivity strengths related to the distance between

6 RF centroids in the source and target location. We hypothesized that functional connectivity

7 strengths would increase with RF overlap for feedforward, but not for feedback networks.

8 To test this, we modeled functional connectivity strength as a function of distance between RF

9 centroids for all recording locations (areas and layers, see Methods). This confirmed the

10 convergent nature of interactions for the gamma band feedforward network $\left(p<.001, R_{a d j}^{2}=\right.$

11 .88), but not for the scale free network $\left(p=.26, R_{a d j}^{2}=.76\right)$ (Figure 5). In addition, this

12 analysis uncovered a divergent pattern of interactions for the infragranular feedback network,

13 with stronger interactions toward more spatially distinct RFs $\left(p<.001, R_{a d j}^{2}=.89\right)$. No RF-

14 dependence was observed for the supragranular feedback network $\left(p=.16, R_{a d j}^{2}=.7\right)$. These

15 results reveal spatial processing differences between the feedforward and feedback networks by

16 showing how the spatial organization of RFs in the source and target location co-determine

17 functional interaction strengths. 

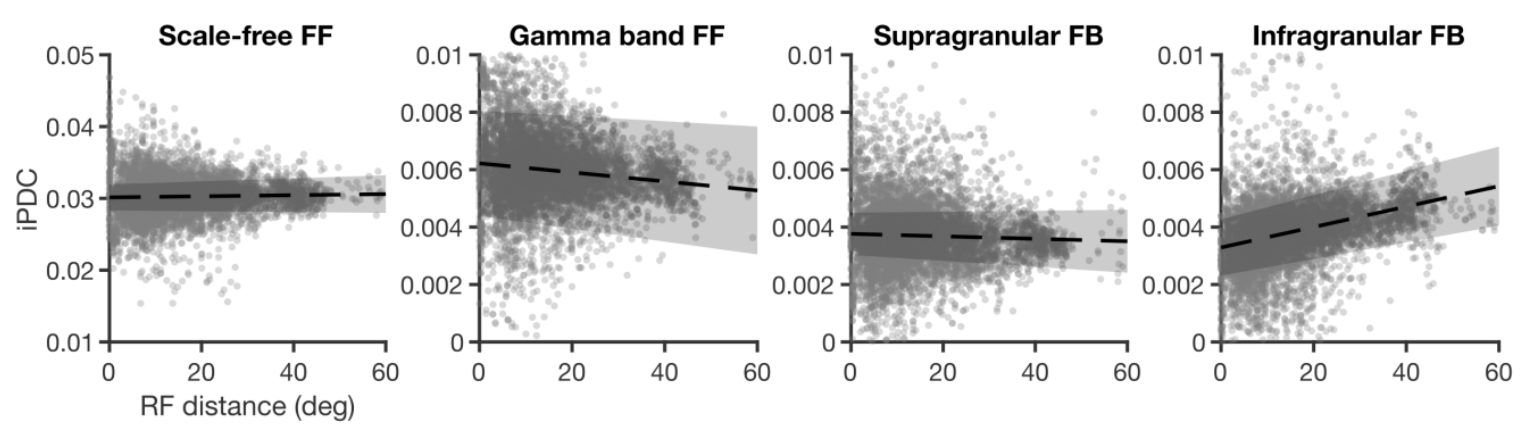

Figure 5. Connectivity as a function of RF distance. Dots represent values acrossss bootstraps, dashed line represents the regressed line and the shaded area indicate the $95 \%$ confidence intervals (on intercept and slope of the model). Data from high contrast condition.

\section{Discussion}

7 We found that functional interactions across visual cortex can be reliably decomposed into four

8 constituent directed networks, based on their distinct laminar connectivity profiles, operational

9 frequencies, temporal dynamics, and hierarchical organizations. The laminar connectivity

10 pathways match anatomical knowledge and provide strong functional evidence for the

11 simultaneous presence of multiple feedforward and feedback streams ${ }^{6,33}$, and extend the notion

12 of functional hierarchies in cortex ${ }^{2}$. We furthermore show distinct functional roles for each of

13 these streams in response to stimuli, reconciling the hierarchical structure of visual cortex with

14 its fast distributed processing.

15 Our analyses demonstrate the existence of two distinct feedforward processing streams. Previous

16 studies have associated feedforward sensory processing with gamma band activity in

17 supragranular layers ${ }^{10,12}$, in line with anatomical connectivity patterns of these layers ${ }^{6,34}$. Our

18 gamma band network provides strong functional support for a feedforward role for layers $2 / 3$ 
1 pathways mediated by gamma band interactions. But in addition, our results indicate a second

2 feedforward stream over infragranular layers that has a scale-free frequency distribution.

3 Evidence of an infragranular feedforward pathway has been previously reported based on

4 anatomical data ${ }^{6}$, but to our best knowledge it has not been reported in functional studies.

5 Our results identify distinct roles in visual processing for the scale-free and the gamma band

6 network. An important clue to the possible function of the scale-free network lies in its power-

7 law frequency distribution. Previous works have related scale-free LFP spectra to overall

8 neuronal population activities ${ }^{45}$, but at a larger-scale, power-law distributions can indicate that a

9 complex system is working near criticality, i.e. at an equilibrium point between two states ${ }^{18}$.

10 Dynamics near a critical point can allow neural activity states to rapidly adjust to changes in the

11 external environment, by providing a flexible mid-level synchronization between neuronal

12 rhythms and fast transition between states via phase resetting ${ }^{46}$. In our data, the scale-free

13 network was the dominant network (strongest amplitude), suggesting its relative importance and

14 a possible role in facilitating the state and activity changes of other frequency-specific networks

15 in response to visual stimulation, an interpretation that is supported by its strong but transient

16 response to visual stimuli, and its lack of RF convergence. In line with such a dynamic resetting

17 role, the scale-free network also showed low autocorrelation in time, and non-convergent RF

18 projections of its upstream interactions, indicating nonspatial processing.

19 The gamma band network, by contrast, responded to visual stimuli with a sustained amplitude

20 increase throughout stimulus presentation. This suggests a role in processing stimulus content

21 that is supported by its sensitivity to stimulus contrast, both in the time and frequency domain. In

22 addition, the RF convergence of its interactions make it suited to process spatial stimulus aspects,

23 because convergent interactions preserve spatial position while propagating induced activity up 
1 the hierarchy. The peak frequency and amplitude of the induced gamma oscillations are

2 previously reported to be modulated by stimulus properties such as contrast, size and location

311,16 , as well as by selective attention ${ }^{47}$. Overall, it has been shown that salient or attended

4 stimuli induce faster and stronger gamma oscillations in early visual areas, and when competing

5 gamma oscillations exist within low-level areas, the faster oscillations have a higher chance to

6 induce activity in higher-level areas through phase coding 13,25. Therefore, the stimulus

7 sensitivity of gamma oscillations has been described as an implementation of bottom-up

8 attentional selection ${ }^{13}$. In our results, the contrast sensitivity of gamma peak and amplitude

9 indicate that elementary forms of such an effect might exist in mice.

10 We found strong evidence for a theta-band modulation of the feedforward networks. This theta

11 modulation seems unlikely to be caused by the feedback networks, because they did not show a

12 sustained response, whereas the theta modulation persisted over the whole period of stimulus

13 presentation (Figure 4). Given its contrast dependency, a cortical origin seems the most likely.

14 Cortical theta activity in sensory areas has been linked to feedforward interactions ${ }^{12,17}$, and plays

15 a role in rhythmic perceptual sampling in monkey and human ${ }^{41,48}$. A proposed mechanism of

16 perceptual sampling is based on theta-gamma cross-frequency coupling, where theta modulates

17 feedforward activity through resynchronization of gamma oscillations between lower and higher

18 visual areas by resetting of the gamma phase ${ }^{13,42}$. Our results show that entire feedforward

19 networks can be modulated by a theta rhythm and provide first evidence for a rhythmic visual

20 sampling in mouse. If behaviorally verified, this means that rhythmic visual sampling is an

21 evolutionary well-preserved feature, and that the causal investigations possible in mice can be

22 employed to help further understand this phenomenon. 
1 We uncovered two feedback networks that operate at alpha and theta frequencies ${ }^{10,12}$. Yet each

2 feedback network showed distinct laminar connectivity, functional hierarchy, contrast sensitivity

3 and relationship with RF distances (Figure 3), suggesting a functional dissociation between

4 feedback streams for stimulus-dependent spatially-organized feedback and global modulatory

5 feedback ${ }^{8}$. The infragranular feedback stream showed a particular contrast sensitivity, with fast

6 amplitude decreases for high contrast, and slower but amplitude increases for low contrast

7 stimuli. In addition, its connectivity pattern was spatially organized, with stronger connections to

8 locations with nonoverlapping RFs. This organization is compatible with a role in contextual

9 spatial processing, such as surround suppression and figure-ground segmentation ${ }^{4,10,44}$, operating

10 over infragranular layers in stimulus-specific ways ${ }^{49}$. It has been shown that feedback over

11 infragranular layers can affect local processes in the cortical column through upward propagation

$12 \quad 31,37$. Likewise, feedback at supragranular layers can modulate activity throughout the column ${ }^{37}$.

13 In our data, the supragranular feedback stream showed no spatial organization of connections,

14 and less sensitivity to contrast, making it suitable for global or non-retinotopic feedback

15 modulations ${ }^{50}$. In line with this characterization, the lowest areas in its hierarchy were motion-

16 sensitive areas AM, LM and RL ${ }^{32}$.

17 Feedback processing is generally thought to arise after a first feedforward sweep, in a two-stage

18 process ${ }^{9}$. Our results show partial support for such a sequential view, in that infragranular

19 feedback increased at longer latencies $(150 \mathrm{~ms})$, and selectively for low contrast stimuli where

20 feedback is expected to play a greater role ${ }^{21}$. In addition, however, our results show that stimuli

21 can evoke quick feedback processing over the supragranular stream, and that they can also

22 quickly suppress ongoing feedback processes. Suppression of theta and alpha feedback due to

23 attention has been previously reported ${ }^{42}$. Taken together, our results characterize visual-evoked 
1 responses as a dynamic re-weighting of feedforward and feedback processes, which are better

2 separable in terms of their functional hierarchies, laminar connectivity and operational

3 frequencies, than they are in time.

4 Overall, feedback amplitudes were small compared to the feedforward amplitudes ${ }^{35}$, and while

5 feedback was transient, feedforward networks showed more robust evoked responses and for

6 longer time. Anatomical studies in monkey show that feedback projections are more numerous

7 than feedforward projections, but that feedforward projections are stronger, especially at shorter

8 range ${ }^{6}$. Our results confirm this with functional evidence and characterize the evoked response

9 as a predominantly feedforward process. But even though the stimuli and task were not

10 optimized for investigating typical roles attributed to feedback connections, like figure-ground

11 segregation ${ }^{4,21}$ or contour integration ${ }^{3}$, the demonstrated presence of two feedback streams in

12 awake but passive mice points to a fundamental importance for feedback in vision. It also reveals

13 similarities between the large-scale functional organization of processing streams in mouse and

14 primates which open up new avenues for investigating large-scale cortical function using causal

15 manipulations. 


\section{Methods}

\section{$2 \quad$ Dataset and visual stimuli}

3 We used publicly available recordings from the Visual Coding - Neuropixels dataset, provided

4 by the Allen Institute for Brain Science ${ }^{2}$. This dataset contains LFPs recorded simultaneously in

54 to 6 visual areas of awake mice, using Neuropixel probes ( ${ }^{51}, 40 \mu \mathrm{m}$ distance between

6 recording channels), with $2.5 \mathrm{KHz}$ sampling rate. Full details on surgery, stimulation protocols

7 and recording techniques are available in the technical white paper 'Allen Brain Observatory -

8 Neuropixels Visual Coding', 2019 (portal.brain-map.org/explore/circuits/visual-coding-

9 neuropixels). The data was downloaded in the format of raw Neurodata Without Borders

10 (NWB), using Allen SDK 1.2.0 (2019).

11 We used a subset of the Brain Observatory stimulus set with a large number of trials, the

12 Functional Connectivity stimulus set. From 14 available LFP recordings in wild type mice, we

13 selected 11 for analysis (average age $=126 \pm 10$ days, 10 males) that showed clear source and

14 sink patterns of current source density (CSD) and had simultaneous recordings from at least four

15 separate areas (Figure 1a). These areas were V1 (recordings from 11 animals), AL (10), RL (9),

16 PM (7), LM (6), and AM (11) of the left hemisphere; 2 animals had simultaneous recordings in 6

17 areas, 6 in 5 and 3 in 4.

18 From the standardized battery of visual stimuli ${ }^{52}$, we selected drifting gratings for their reliably

19 evoked signals and high number of trials (75 trials for each grating orientation and contrast).

20 Gratings were presented in random order, with a high $(80 \%)$ or low $(10 \%)$ contrast at detectable

21 levels ${ }^{53}$, four different orientations $(0,45,90$ or 135 degrees), and with a spatial frequency of 
10.04 cycles/deg and temporal frequency of $2 \mathrm{~Hz}$ (drifting left-to-right). Stimuli were presented

2 monocularly to the right eye on a monitor that covered $120 \times 95$ degrees of visual angle. The

3 paradigm consisted of two seconds of stimulus presentation, and one second of inter-stimulus

$4 \quad$ interval during which a uniform mean luminance gray was presented.

\section{$5 \quad$ Preprocessing and layer assignment}

6 For each trial, we considered the epoch from $300 \mathrm{~ms}$ before to $1000 \mathrm{~ms}$ after stimulus onset. We

7 pooled the epochs from four orientations together which resulted in 300 epochs per animal, per

8 contrast.

9 To identify representative electrodes for each cortical layer (L1 to L6) we derived CSD patterns

10 from averaged LFP data ${ }^{54}$. We assigned layers based on sources and sinks across cortical depth,

11 following ${ }^{40}$, taking into account depth estimates provided with the dataset. The average cortical

12 depths of selected electrodes for each layer are listed in Table 1.

13 Table 1. Average cortical depth of selected channels for each layer and area (mean \pm standard deviation)

\begin{tabular}{|l|l|l|l|l|l|l|}
\hline & V1 & LM & RL & AL & PM & AM \\
\hline L1 & $80 \pm 25$ & $87 \pm 39$ & $76 \pm 13$ & $73 \pm 24$ & $80 \pm 0$ & $80 \pm 25$ \\
\hline L2 & $160 \pm 25$ & $167 \pm 39$ & $156 \pm 13$ & $149 \pm 31$ & $183 \pm 31$ & $164 \pm 28$ \\
\hline L3 & $255 \pm 32$ & $253 \pm 41$ & $244 \pm 13$ & $233 \pm 35$ & $297 \pm 31$ & $269 \pm 40$ \\
\hline L4 & $407 \pm 39$ & $400 \pm 51$ & $360 \pm 35$ & $356 \pm 52$ & $451 \pm 30$ & $407 \pm 50$ \\
\hline L5 & $542 \pm 55$ & $560 \pm 51$ & $511 \pm 52$ & $495 \pm 60$ & $583 \pm 31$ & $545 \pm 48$ \\
\hline L6 & $785 \pm 70$ & $767 \pm 39$ & $733 \pm 45$ & $720 \pm 82$ & $777 \pm 39$ & $764 \pm 55$ \\
\hline
\end{tabular}

14

15 In order to remove the common reference, which could cause spurious estimation of functional

16 connectivity, we applied bipolar re-referencing by computing the difference of the LFPs from 
1 two neighboring channels ${ }^{55,56}$. LFP data were downsampled to $250 \mathrm{~Hz}$ after anti-aliasing

2 filtering.

\section{$3 \quad$ Functional connectivity}

4 To estimate time- and frequency-resolved directed functional connectivity we used a multivariate

5 parametric approach, the Self-Tuning Optimized Kalman filter (STOK) ${ }^{23}$. STOK uses a Kalman

6 filter formulation that is optimized to model rapidly fluctuating between-signal dependencies

7 under unknown noise conditions, resulting in a time-varying multivariate autoregressive

8 (tvMVAR) model. To estimate the tvMVAR model, a model order parameter $p$ should be

9 estimated prior to analysis. This parameter indicates how much of the past information should be

10 included in estimation of current state. Here we selected the optimal model order by minimizing

11 the difference between the tvMVAR model power spectra and the spectra calculated by a

12 nonparametric method based on complex Morlet Wavelet ${ }^{57}$, for each animal separately. We then

13 selected the maximum optimal order observed over animals ( $p=15$ samples, or $60 \mathrm{~ms}$ ) as the

14 model order, and calculated tvMVAR models per animal and condition (low, high contrast)

15 using all single trial epochs and a filter factor of 0.98 . STOK code is freely available for Matlab

16 (https://github.com/PscDavid/dynet_toolbox) and Python (https://github.com/joanrue/pydynet).

17 After Fourier transformation, tvMVAR coefficients were normalized to express a directed

18 measure of functional connectivity in line with Granger causality, the information partial

19 directed coherence (iPDC) ${ }^{22,59,60}$. Resulting iPDC matrices contained the layer-specific directed

20 functional connectivity between all recorded areas, for every frequency between 1 and $100 \mathrm{~Hz}(1$

$21 \mathrm{~Hz}$ resolution), and timepoint between -300 to $1000 \mathrm{~ms}$ around stimulus onset (4 ms resolution).

22 We preserved the between-area connections for further analysis, averaged across animals, and 
1 unfolded the resulting connectivity matrix into 5 dimensions as follows: source layers, target

2 layers, time, frequency, and between-area connections (30 directed connections between the 6

3 areas).

\section{$4 \quad$ Parallel Factor Analysis}

5 PARAFAC decomposes a multiway matrix into a fixed number of components, with each

6 component represented by a set of loading vectors that correspond to the original data

7 dimensions 26,61 . We applied PARAFAC to the averaged 5-dimensional connectivity matrices.

8 The resulting PARAFAC decomposition can be expressed as:

$$
i P D C_{s l, t l, t, f, c r}=\sum_{k=1}^{K} a_{s l, k} \cdot b_{t l, k} \cdot c_{t, k} \cdot d_{f, k} \cdot e_{c, k}
$$

10 Where $\mathrm{K}$ is the number of components, and $\boldsymbol{a}_{\boldsymbol{s}, \boldsymbol{k}}, \boldsymbol{b}_{t \boldsymbol{t}, \boldsymbol{k}}, \boldsymbol{c}_{\boldsymbol{t}, \boldsymbol{k}}, \boldsymbol{d}_{\boldsymbol{f}, \boldsymbol{k}}$ and $\boldsymbol{e}_{\boldsymbol{c}, \boldsymbol{k}}$ correspond to the

11 loading vectors for component $k$, for each source layer, target layer, time point, frequency point,

12 and between-area connection. Loading vectors were estimated using alternating least square with

13 random initialization and a non-negativity constraint. The variance of the data is usually kept in

14 one of the loading vectors of PARFAC model and the other loading vectors are normalized to

15 have variance of 1 . In our case, the variance was kept in the temporal loadings. We used the N-

16 way Matlab toolbox for PARAFAC decomposition ${ }^{62}$.

17 To derive the most informative and valid PARAFAC model, an appropriate number of

18 components needs to be selected. With too few components, the true underlying components of

19 the data cannot be extracted, while with too many, the results will contain correlated components

20 that do not represent the underlying variables. To identify the appropriate number of 
1 components, we combined multiple indicators of model quality and visual inspection of the

2 resulting components, following previous works ${ }^{26-30}$. To exclude the possibility that component

3 selection was driven by data from a subset of animals, we first created bootstrapped averages by

4 randomly selecting 8 (out of 11 ) animals. For each bootstrapped average $(n=10)$, we calculated

5 Corcondia scores, variance explained, mean squared error, and model convergence. Using these

6 scores across bootstraps we found that models with 4 components were the best choice for our

7 data: they substantially increased model fit as compared to sparser models, while including more

8 components resulted in high correlations between some of them.

9 Finally, to verify the reliability of the PARAFAC model across animals, we bootstrapped iPDC

10 averages $(n=500$, random selection as above) and extracted the four components for each

11 bootstrap. We checked component consistency across bootstraps by calculating the pairwise

12 correlations between the loading vectors of PARAFAC components, resulting in intra- and inter-

13 component consistency values.

\section{Statistical analysis on PARAFAC loadings}

15 To specify the characteristic of each network, we investigated the loading vectors of the

16 PARAFAC models using statistical analysis. To indicate the source and target layers that

17 contribute most to each network, we compared the loading values of each source/target layer

18 over bootstraps to uniform distribution of weights over layers using t-test. Uniform distribution

19 indicates no laminar specificity for the network, while layers with loading values larger than the

20 uniform distribution have significant contribution to that network.

21 For temporal loadings, we determined significant evoked responses from baseline by comparing

22 the temporal loading values of each post-stimulus time point with averaged pre-stimulus 
1 temporal loading values, using t-test and Bonferroni correction. For visualization purpose, we

2 converted the temporal loadings to percentage of change by subtracting and then dividing each

3 temporal loading by its averaged pre-stimulus loading values.

4 Since the variance of the data is preserved in the temporal loadings, we indicated the relative

5 overall amplitude of each PARAFAC component by dividing its averaged temporal loading by

6 the sum of averaged temporal loadings of all components.

\section{$7 \quad$ Model comparison of frequency distributions}

8 To characterize frequency distribution of the PARAFAC components, we fitted three

9 distributions to the frequency loadings in each bootstrap separately. Power-law (in the form of

$\left.10 \quad c . f^{-\beta}\right)$, lognormal $(\log N(\mu, \sigma))$, and exponential $\left(c . e^{\beta . f}\right)$ distributions were fitted using nlinfit

11 function in Matlab and the mean squared error for each fitting was calculated. The model with

12 the lowest average mean squared error over the bootstraps was considered as the best model fit.

13 We also fitted the same models to the power spectrum density of LFPs, calculated from the AR

14 coefficients, for each bootstrap and each ROI separately and used mean squared error to indicate

15 the best model fit.

\section{Functional hierarchy analysis}

17 We used loading vectors of between-area connectivity strengths to calculate hierarchy scores,

18 employing a method specifically proposed for directed functional connectivity strengths ${ }^{12}$. First,

19 the directed influence asymmetry index $\left(\mathrm{DAI}_{\mathrm{ij}}\right)$ was estimated based on between-area loading

20 vector with element $\boldsymbol{e}_{\boldsymbol{i}}$ indicating the connection from area $j$ to $i$. 
$1 \quad D A I_{i j}=\frac{e_{i j}-e_{j i}}{e_{i j}+e_{j i}}$

2 We scaled DAIs to the range [-2.5 2.5] in order to allow 6 levels of hierarchy (considering 6

3 areas). Then, for each target area, we shifted the DAIs from all source areas so that the smallest

4 value was 1 . Then we averaged the rescaled and shifted DAIs $(\widehat{D A} I)$ for each source to estimate

5 the hierarchy score of the source area as $H_{i}=\sum_{j} \widehat{D A} I_{i j}$.

\section{$6 \quad$ Receptive field analysis}

7 The significant receptive fields (RF) were calculated based on a permutation-based method used

8 in ${ }^{2}$ and using the strength of evoked LFPs in a Gabor stimuli condition in the dataset. RF

9 distances were calculated as the Euclidean distance between the centroids of the significant RFs.

10 We only considered the between-area connections which had source and target areas with

11 significant receptive fields $(80 \%)$.

12 To examine the relationship between RF distance and functional connectivity strengths, we

13 reconstructed between-area connectivity strengths from the PARAFAC results using Kronecker

14 products of the loading vectors, for each component and bootstrap separately. We then averaged

15 the reconstructed matrix over time, frequency and layers to obtain a single connectivity value for

16 each between-area connection.

17 Using linear mixed-effect regression, we modelled connectivity strengths as a function of the

18 continuous predictor RF distance, including bootstrap and between-area connection as random

19 effects over the intercept, to account for between-area and animal variability respectively. This

20 model was preferred over a simpler fixed-effects model without random effects, as shown by a 
1 Likelihood Ratio Test. Modeling was done using the fitlme function with maximum likelihood

2 estimation in Matlab.

\section{Acknowledgement}

5 This research was supported by the Swiss National Science Foundation (grants PP00P1_157420

6 and PP00P1_183714).

\section{Author contributions}

9 EB and GP: Investigation, Methodology, Writing; EB: Data curation, Formal Analysis,

10 Visualization; GP: Conceptualization, Funding acquisition, Supervision.

\section{Competing Interests statement}

13 The authors declare no competing financial or non-financial interests. 


\section{References}

2

3

4

5

1. Bullier, J. Integrated model of visual processing. Brain Res. Rev. 36, 96-107 (2001).

2. Siegle, J. H. et al. A survey of spiking activity reveals a functional hierarchy of mouse corticothalamic visual areas. bioRxiv 805010 (2019) doi:https://doi.org/10.1101/805010.

3. Chen, M. et al. Incremental Integration of Global Contours through Interplay between Visual Cortical Areas. Neuron 82, 682-694 (2014).

4. Poort, J., Self, M. W., Vugt, B. van, Malkki, H. \& Roelfsema, P. R. Texture Segregation Causes Early Figure Enhancement and Later Ground Suppression in Areas V1 and V4 of Visual Cortex. Cereb. Cortex (2016) doi:10.1093/cercor/bhw235.

5. Felleman, D. J. \& Van Essen, D. C. Distributed Hierarchical Processing in the Primate. Cereb. Cortex 1, 1-47 (1991).

6. Markov, N. T. et al. Anatomy of hierarchy: feedforward and feedback pathways in macaque visual cortex. J. Comp. Neurol. 522, 225-259 (2014).

7. Harris, J. A. et al. Hierarchical organization of cortical and thalamic connectivity. Nature 575, 195-202 (2019).

8. Gilbert, C. D. \& Li, W. Top-down influences on visual processing. Nat. Rev. Neurosci. 14, $350-363$ (2013).

9. Lamme, V. A. F. \& Roelfsema, P. R. The distinct modes of vision offered by feedforward and recurrent processing. Trends Neurosci. 23, 571-579 (2000).

10. Van Kerkoerle, T. et al. Alpha and gamma oscillations characterize feedback and feedforward processing in monkey visual cortex. Proc. Natl. Acad. Sci. 111, 14332-14341 (2014). 
1 11. Henrie, J. A. \& Shapley, R. LFP power spectra in V1 cortex: the graded effect of stimulus contrast. J. Neurophysiol. 94, 479-490 (2005).

3 12. Bastos, A. M. et al. Visual areas exert feedforward and feedback influences through distinct

4 frequency channels. Neuron 85, 390-401 (2015).

5 13. Fries, P. Rhythms for cognition: communication through coherence. Neuron 88, 220-235

$6 \quad(2015)$.

7 14. von Stein, A. \& Sarnthein, J. Different frequencies for different scales of cortical integration:

8 from local gamma to long range alpha/theta synchronization. Int. J. Psychophysiol. 38, 301-

$9313(2000)$.

15. Wang, X.-J. Neurophysiological and Computational Principles of Cortical Rhythms in

11 Cognition. Physiol. Rev. 90, 1195-1268 (2010).

12 16. Ray, S. \& Maunsell, J. H. Differences in gamma frequencies across visual cortex restrict

13 their possible use in computation. Neuron 67, 885-896 (2010).

14 17. Kienitz, R. et al. Theta, but Not Gamma Oscillations in Area V4 Depend on Input from

15 Primary Visual Cortex. Curr. Biol. (2020) doi:10.1016/j.cub.2020.10.091.

16 18. Chialvo, D. R. Emergent complex neural dynamics. Nat. Phys. 6, 744-750 (2010).

17 19. He, B. J., Zempel, J. M., Snyder, A. Z. \& Raichle, M. E. The temporal structures and

18 functional significance of scale-free brain activity. Neuron 66, 353-369 (2010).

19 20. Carandini, M. \& Heeger, D. J. Summation and division by neurons in primate visual cortex.

$20 \quad$ Science 264, 1333-1336 (1994).

21 21. Hupé, J. M. et al. Cortical feedback improves discrimination between figure and background

22 by V1, V2 and V3 neurons. Nature 394, 784-787 (1998). 
1 22. Takahashi, D. Y., Baccalá, L. A. \& Sameshima, K. Information theoretic interpretation of

2 frequency domain connectivity measures. Biol. Cybern. 103, 463-469 (2010).

3 23. Pascucci, D., Rubega, M. \& Plomp, G. Modeling time-varying brain networks with a self-

$4 \quad$ tuning optimized Kalman filter. PLOS Comput. Biol. 16, e1007566 (2020).

5 24. Roberts, M. J. et al. Robust gamma coherence between macaque V1 and V2 by dynamic

6 frequency matching. Neuron 78, 523-536 (2013).

7 25. Lowet, E. et al. Input-Dependent Frequency Modulation of Cortical Gamma Oscillations

8 Shapes Spatial Synchronization and Enables Phase Coding. PLOS Comput. Biol. 11,

$9 \quad$ e1004072 (2015).

10 26. Bro, R. PARAFAC. Tutorial and applications. Chemom. Intell. Lab. Syst. 38, 149-172

$11 \quad$ (1997).

12 27. Barzegaran, E., Vildavski, V. Y. \& Knyazeva, M. G. Fine structure of posterior alpha rhythm

13 in human EEG: Frequency components, their cortical sources, and temporal behavior. Sci.

$14 \quad$ Rep. 7, 8249 (2017).

15 28. Miwakeichi, F. et al. Decomposing EEG data into space-time-frequency components using

16 Parallel Factor Analysis. NeuroImage 22, 1035-1045 (2004).

17 29. Pester, B., Ligges, C., Leistritz, L., Witte, H. \& Schiecke, K. Advanced Insights into

18 Functional Brain Connectivity by Combining Tensor Decomposition and Partial Directed

19 Coherence. PLoS ONE 10, (2015).

20 30. Bro, R. \& Kiers, H. A. A new efficient method for determining the number of components in

21 PARAFAC models. J. Chemom. J. Chemom. Soc. 17, 274-286 (2003). 
1 31. Plomp, G., Quairiaux, C., Kiss, J. Z., Astolfi, L. \& Michel, C. M. Dynamic connectivity among cortical layers in local and large-scale sensory processing. Eur. J. Neurosci. 40, $3215-3223$ (2014).

4 32. Marshel, J. H., Garrett, M. E., Nauhaus, I. \& Callaway, E. M. Functional specialization of $5 \quad$ seven mouse visual cortical areas. Neuron 72, 1040-1054 (2011).

6 33. Markov, N. T. et al. Cortical High-Density Counterstream Architectures. Science 342, $7 \quad 1238406(2013)$.

8 34. D’Souza, R. D. \& Burkhalter, A. A Laminar Organization for Selective Cortico-Cortical 9 Communication. Front. Neuroanat. 11, (2017).

11 Top-Down Generative Networks. NeuroImage 117479 (2020).

12 36. von Stein, A., Chiang, C. \& König, P. Top-down processing mediated by interareal

13 synchronization. Proc. Natl. Acad. Sci. 97, 14748-14753 (2000).

14 37. Larkum, M. E., Zhu, J. J. \& Sakmann, B. A new cellular mechanism for coupling inputs arriving at different cortical layers. Nature 398, 338-341 (1999).

16 38. Murayama, M. et al. Dendritic encoding of sensory stimuli controlled by deep cortical 17 interneurons. Nature 457, 1137-1141 (2009).

18 39. Takahashi, N., Oertner, T. G., Hegemann, P. \& Larkum, M. E. Active cortical dendrites modulate perception. Science 354, 1587-1590 (2016). 
1 41. Kienitz, R. et al. Theta Rhythmic Neuronal Activity and Reaction Times Arising from

$2 \quad$ Cortical Receptive Field Interactions during Distributed Attention. Curr. Biol. 28, 2377-

$3 \quad 2387 . e 5(2018)$.

4 42. Spyropoulos, G., Bosman, C. A. \& Fries, P. A theta rhythm in macaque visual cortex and its

5 attentional modulation. Proc. Natl. Acad. Sci. 115, E5614-E5623 (2018).

6 43. Liebe, S., Hoerzer, G. M., Logothetis, N. K. \& Rainer, G. Theta coupling between V4 and

7 prefrontal cortex predicts visual short-term memory performance. Nat. Neurosci. 15, 456-

$8 \quad 462(2012)$.

9 44. Zipser, K., Lamme, V. A. F. \& Schiller, P. H. Contextual Modulation in Primary Visual

Cortex. J. Neurosci. 16, 7376-7389 (1996).

11 45. Manning, J. R., Jacobs, J., Fried, I. \& Kahana, M. J. Broadband shifts in local field potential

12 power spectra are correlated with single-neuron spiking in humans. J. Neurosci. 29, 13613-

$13 \quad 13620(2009)$.

14 46. Palva, S. \& Palva, J. M. Roles of Brain Criticality and Multiscale Oscillations in Temporal

15 Predictions for Sensorimotor Processing. Trends Neurosci. 41, 729-743 (2018).

16 47. Bosman, C. A. et al. Attentional stimulus selection through selective synchronization

17 between monkey visual areas. Neuron 75, 875-888 (2012).

18 48. Busch, N. A. \& VanRullen, R. Spontaneous EEG oscillations reveal periodic sampling of

19 visual attention. Proc. Natl. Acad. Sci. 107, 16048-16053 (2010).

20 49. Vélez-Fort, M. et al. The stimulus selectivity and connectivity of layer six principal cells

21 reveals cortical microcircuits underlying visual processing. Neuron 83, 1431-1443 (2014).

22 50. Harris, K. D. \& Mrsic-Flogel, T. D. Cortical connectivity and sensory coding. Nature 503, $23 \quad 51-58(2013)$. 
1 51. Jun, J. J. et al. Fully integrated silicon probes for high-density recording of neural activity.

2 Nature 551, 232-236 (2017).

52. de Vries, S. E. J. et al. A large-scale standardized physiological survey reveals functional organization of the mouse visual cortex. Nat. Neurosci. 23, 138-151 (2020).

53. Histed, M. H., Carvalho, L. A. \& Maunsell, J. H. R. Psychophysical measurement of contrast sensitivity in the behaving mouse. J. Neurophysiol. 107, 758-765 (2011).

54. Mitzdorf, U. Current source-density method and application in cat cerebral cortex: investigation of evoked potentials and EEG phenomena. Physiol. Rev. 65, 37-100 (1985).

55. Trongnetrpunya, A. et al. Assessing Granger Causality in Electrophysiological Data: Removing the Adverse Effects of Common Signals via Bipolar Derivations. Front. Syst. Neurosci. 189 (2016) doi:10.3389/fnsys.2015.00189.

56. Plomp, G., Larderet, I., Fiorini, M. \& Busse, L. Layer 3 Dynamically Coordinates Columnar Activity According to Spatial Context. J. Neurosci. 39, 281-294 (2019).

57. Pagnotta, M. F., Dhamala, M. \& Plomp, G. Benchmarking nonparametric Granger causality: Robustness against downsampling and influence of spectral decomposition parameters. NeuroImage 183, 478-494 (2018).

58. Pascucci, D., Rubega, M. \& Plomp, G. Modeling time-varying brain networks with a selftuning optimized Kalman filter. bioRxiv 856179 (2019) doi:doi: https://doi.org/10.1101/856179.

59. Granger, C. W. J. Investigating Causal Relations by Econometric Models and Cross-Spectral Methods. Econometrica 37, 424-38 (1969).

60. Baccalá, L. A. \& Sameshima, K. Partial directed coherence: a new concept in neural structure determination. Biol. Cybern. 84, 463-474 (2001). 
1 61. Harshman, R. A. Foundations of the PARAFAC procedure: Models and conditions for an"

2 explanatory" multimodal factor analysis. (1970).

3 62. Andersson, C. A. \& Bro, R. The N-way toolbox for MATLAB. Chemom. Intell. Lab. Syst.

$4 \quad 52,1-4(2000)$.

5

6 